\title{
In Vivo Fiber Photometry Reveals Signature of Future Stress Susceptibility in Nucleus Accumbens
}

\author{
Jessie Muir', Zachary S Lorsch ${ }^{2}$, Charu Ramakrishnan ${ }^{3}$, Karl Deisseroth $^{3}$, Eric J Nestler ${ }^{2}$, Erin S Calipari ${ }^{2}$ and \\ Rosemary C Bagot ${ }^{*, 1,4}$
}

'Department of Psychology, McGill University, Montreal, QC, Canada; ${ }^{2}$ Fishberg Department of Neuroscience and Friedman Brain Institute, Icahn School of Medicine at Mount Sinai, New York, NY, USA; ${ }^{3}$ Department of Bioengineering, Stanford University, Stanford, CA, USA; ${ }^{4}$ Ludmer Centre for Neuroinformatics and Mental Health Montreal, QC, Canada

\begin{abstract}
Recognizing why chronic stress causes only a subset of individuals to become depressed is critical to understanding depression on a basic level and, also, to developing treatments that increase resilience. Stress-induced alterations in the activity of reward-related brain regions, such as the nucleus accumbens (NAc), are linked to the pathophysiology of depression. However, it has been difficult to determine if differences in stress susceptibility are pre-existing or merely an effect of chronic stress. The NAc consists largely of medium spiny neurons (MSNs), distinguished by their predominant expression of either DI or D2 dopamine receptors. Mice that develop depressive-like symptoms after chronic social defeat stress show distinct changes in the activity of these two cell subtypes. Until now it has not been possible to determine whether such effects are merely a consequence of stress or in fact precede stress and, thus, have utility in preidentifying stress-susceptible individuals. The goal of this study was to define a cell-type specific signature of stress susceptibility and resilience. Using fiber photometry calcium imaging, we recorded calcium transients in NAc D I- and D2-MSNs in awake behaving mice and found that DI-MSN activity is a predictive marker of depression susceptibility: prior to stress, mice that will later become resilient had increased baseline DI - MSN activity, and increased calcium transients specific to social interaction. Differences in D2- MSN activity were not specific to social interaction. Our findings identify a pre-existing mechanism of stress-induced susceptibility, creating the potential to target preventative interventions to the most relevant populations.

Neuropsychopharmacology (2018) 43, 255-263; doi:I0.1038/npp.2017.122; published online 5 July 2017
\end{abstract}

\section{INTRODUCTION}

Epidemiological studies highlight the importance of stress in the etiology of depression (Kendler et al, 1999; Kessler, 1997). However, in reality only a minority of people who experience stress become depressed. Identifying the neural mechanisms that increase risk for depression is of fundamental importance to both treatment and prevention. The chronic social defeat stress (CSDS) paradigm captures variation in stress susceptibility; genetically inbred mice exposed to the same experience phenotypically diverge: susceptible mice exhibit depressive-like symptoms such as anhedonia and decreased social interaction, whereas resilient mice fail to show these changes (Berton et al, 2006; Krishnan et al, 2007). Animal models of depression, such as CSDS, have provided important insights into key neuronal changes associated with depression-like states (Bagot et al, 2015; Berton et al, 2006; Chaudhury et al, 2013; Covington et al, 2010; Dias et al, 2014; Francis et al, 2015; Krishnan et al, 2007; Lim et al,

\footnotetext{
*Correspondence: Dr RC Bagot, Department of Psychology, McGill University, Stewart Biology Bldg, N8/4, 1205 Ave. Dr Penfield, Montréal, Canada H3A IBI, Tel: +I $5 \mid 4398$ 34|9, Fax: $5 \mid 4398$ 3255, E-mail: rosemary.bagot@mcgill.ca

Received 24 March 2017; revised 19 May 2017; accepted 23 May 20 17; accepted article preview online 7 June 2017
}

2012; Lobo et al, 2013; Sun et al, 2015; Tye et al, 2013; Vialou et al, 2010), however, understanding the neuronal mechanisms that guide the initial development of susceptibility or resilience remains to be understood. One major challenge in identifying risk factors is the lack of prospective mechanistic studies: assessments are made post stress, making it impossible to distinguish stress-induced neuronal plasticity from the intrinsic factors that render an individual susceptible to stress. Understanding the neuronal mechanisms that support susceptibility $v s$ resilience after stress can inform treatment, but identifying pre-existing features that differentiate individuals that will become susceptible and those that will remain resilient when challenged by stress opens the door to prevention.

The nucleus accumbens (NAc) is an important structure in motivation and reward (Schultz, 2006; Wise, 2004). It is comprised predominantly of medium spiny neurons (MSNs) distinguished by their relative expression of D1 or D2 dopamine receptors (Gerfen and Surmeier, 2011). These two cell types have different downstream targets and play opposing roles in motivation, with D1-MSNs implicated in reward and D2-MSNs primarily implicated in aversion (Baik, 2013; Kravitz et al, 2012; Lobo et al, 2010). Balanced activity between these two cell populations supports normal behavior, with selective dysregulation in these cell types 
implicated in the pathophysiology of depression (Dias et al, 2014; Francis et al, 2015; Lim et al, 2012). Differential activity of D1- and D2-MSNs has been observed after stress in susceptible and resilient mice (Francis et al, 2015). Optogenetic activation of D1-MSNs after chronic stress promotes resilience, whereas pharmacological inhibition promotes susceptibility. Furthermore, activation of D2-MSNs promotes susceptibility after acute stress and, following chronic social defeat, excitatory inputs onto D2-MSNs are increased, but decreased onto D1-MSNs, in susceptible mice (Francis et al, 2015). It is not known if differences in D1 and D2 activity emerge only after stress or if pre-existing differences exist that might identify those individuals that will go on to develop depressive-like symptoms in the face of stress.

We hypothesized that pre-existing differences in D1- and D2-MSN activity may have a role in regulating stress susceptibility, ultimately making some mice more susceptible to stress. We aimed to identify the pre-existing endogenous patterns of in vivo neuronal activity from which susceptibility $v s$ resilience emerge. Using fiber photometry (Gunaydin et al, 2014), a novel technique that allows for the measurement of calcium transients from selected cell types in vivo, we measured real-time neuronal activity in NAc D1 and D2-MSNs in awake behaving mice prior to stress and identified pre-stress differences in D1 signaling as a key risk factor for stress susceptibility. We report that resting D1-MSN activity and temporally specific calcium transients during social interaction associate with emergent phenotypes after social defeat, whereas D2-MSN activity appears to play a more complex role in regulating stress susceptibility.

\section{MATERIALS AND METHODS}

\section{Experimental Animals}

Male 8-16 week-old D1-Cre and D2-Cre BAC transgenic mice on C57BL/6 J background mice initially obtained from NINDS/GENSAT (www.gensat.org/index.html) were bred at Icahn School of Medicine at Mount Sinai and 6-month-old CD1-retired breeders were obtained from Jackson labs. All mice were maintained on a 12 -h light-dark cycle at $22-25^{\circ} \mathrm{C}$ with ad libitum access to food and water. D1-Cre and D2-Cre mice were group housed five per cage until the start of defeat. A total of 21 mice were included in this study: 9 D1-cre transgenic mice and 12 D2-cre transgenic mice. All experimental manipulations occurred during the light cycle. All experiments were conducted in accordance with guidelines of Mount Sinai's Animal Care and Use Committee.

\section{Stereotaxic Fiber Implantation and Virus Injection}

To achieve cell-type specific GCaMP6f expression, D1-Cre and D2-Cre were injected with AAVdj-EF1 $\alpha$-DIOGCaMP6f-WPRE virus $\left(5 \times 10^{-12} / \mathrm{ml}\right)$. Stereotaxic surgery was performed under ketamine $(100 \mathrm{mg} / \mathrm{kg}) / x y l a z i n e$ $(10 \mathrm{mg} / \mathrm{kg})$ anesthesia to target the NAc $(\mathrm{A} / \mathrm{P}+1.3, \mathrm{~L} / \mathrm{M}$ $+0.5, \mathrm{D} / \mathrm{V},-4.4 \mathrm{~mm})$ and $0.5 \mu \mathrm{l}$ virus was infused at a rate of $0.1 \mu \mathrm{l}$ per $\mathrm{min}$ and allowed to diffuse for $10 \mathrm{~min}$ before the needle was withdrawn. Chronically implantable optic fibers (Doric Lenses) with $400 \mu \mathrm{m}$ core, $0.48 \mathrm{NA}$ optic fiber threaded through metal ferrules were then implanted above the viral injection site $(\mathrm{A} / \mathrm{P}+1.3, \mathrm{~L} / \mathrm{M}+0.5, \mathrm{D} / \mathrm{V},-4.3)$. Recordings began a minimum 4 weeks after surgery to allow sufficient time for stable and robust virus expression.

\section{CSDS and Social Interaction}

An established CSDS protocol was used (Berton et al, 2006; Golden et al, 2011). In brief, D1-Cre and D2-Cre mice were subjected to 10 daily $5 \mathrm{~min}$ aggressive encounters with a novel CD1 mouse after which they were separated from the CD1 mouse by a plexiglas divide to allow for sensory but not physical contact for the remainder of the $24 \mathrm{~h}$ period. This protocol yields two phenotypes (susceptible and resilient) identified by an animal's social interaction with a novel CD1 mouse in a social interaction test $24 \mathrm{~h}$ after CSDS. Mice were tested in a social interaction test prior to the start of CSDS and again $24 \mathrm{~h}$ after CSDS. Mice were not pre-exposed to the interaction arena before the social interaction tests. In the first $2.5 \mathrm{~min}$ test (no target), mice explore an arena $(44 \times 44 \mathrm{~cm})$ containing a plexiglas mesh enclosure on one wall $(10 \times 6 \mathrm{~cm})$. In the second $2.5 \mathrm{~min}$ test (target), a novel CD1 mouse (social target) is placed in the plexiglas mesh enclosure and mice again explore freely. Time spent in the interaction zone around the plexiglas mesh enclosure $(14 \times 26 \mathrm{~cm})$, and the corner zones $(10 \times 10 \mathrm{~cm})$, was recorded by video tracking software (Ethovision XT, Noldus). To generate precise behavioral time-stamps to align with the neuronal calcium signal, the timing of corner entries and proximal interaction events were annotated by an experimenter blind to condition (Observer XT, Noldus); proximal interaction events were scored when the mouse's nose contacted the mesh grid of the enclosure and corner entries were scored when the mouse's nose crossed into the corner zones.

\section{Fiber Photometry}

This technique allows for the measurement of neuronal calcium transients in real time (Calipari et al, 2016; Gunaydin et al, 2014). Two light emitting diodes at $490 \mathrm{~nm}$ (GCaMP stimulation wavelength) and $405 \mathrm{~nm}$ (control for artifactual fluorescence) (Thorlabs) were reflected off dichroic mirrors (FF495; Semrock) and coupled to a $400 \mu \mathrm{m}$ 0.4 N.A. optical fiber (BFH48-600, Thorlabs) using a $400 \times 0.4$ N.A. microscope objective (Olympus) and fiber launch (Thorlabs). The emission light was collected by the same optical fiber, passed through a GFP filter and focused onto a photodetector (model 2151 femtowatt photoreceiver; Newport) where the two output signals were separated based on modulation frequency. Samples were collected at a frequency of $381 \mathrm{~Hz}$.

Recordings were made from NAc D1- or D2-MSNs in mice during both target $(2.5 \mathrm{~min})$ and no target $(2.5 \mathrm{~min})$ social interaction tests $24 \mathrm{~h}$ prior to the start of the defeat protocol and in the defeat cage (separated from aggressor by plexiglas divider) immediately before $(2 \mathrm{~min})$ and after (5 min) the first aggressive encounter.

Data were extracted and analyzed using custom-written scripts in Matlab R2016b (The MathWorks). To normalize the data, the control channel was fitted to and then subtracted from the raw trace, giving the $\Delta \mathrm{F} / \mathrm{F}$. For peak detection, data were high-pass filtered and transformed to a 
a

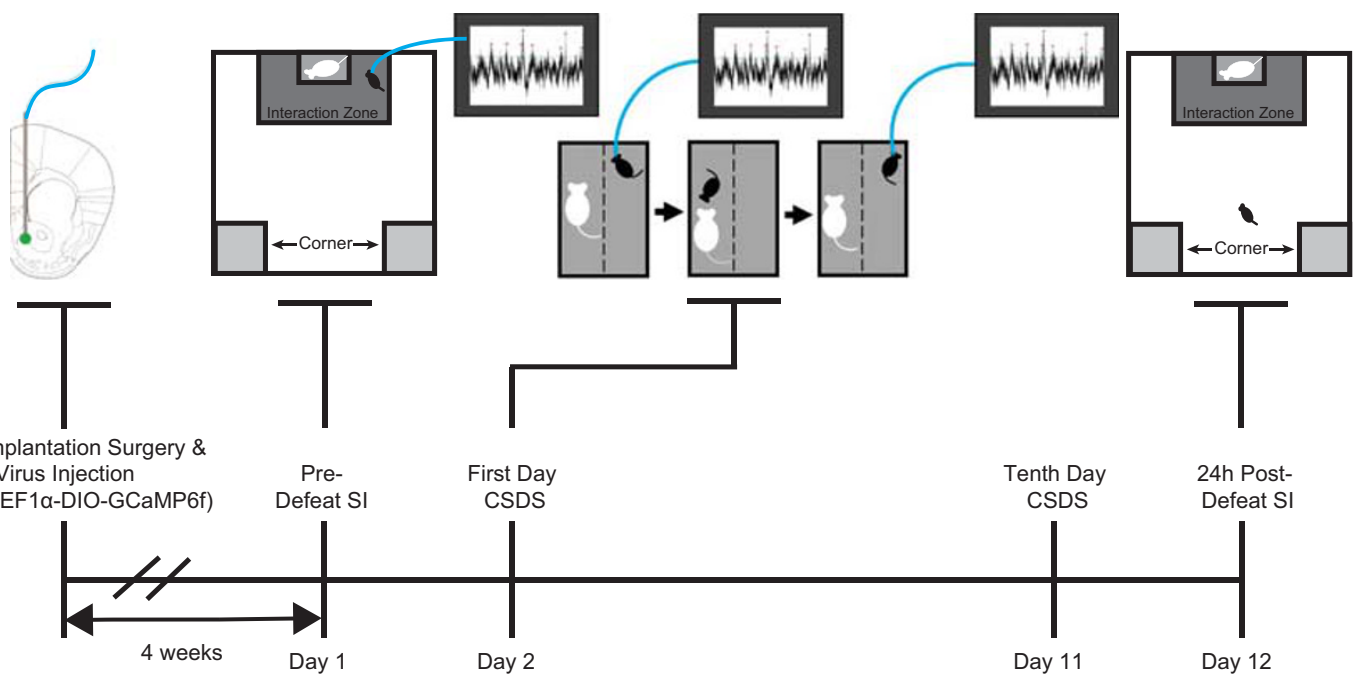

b
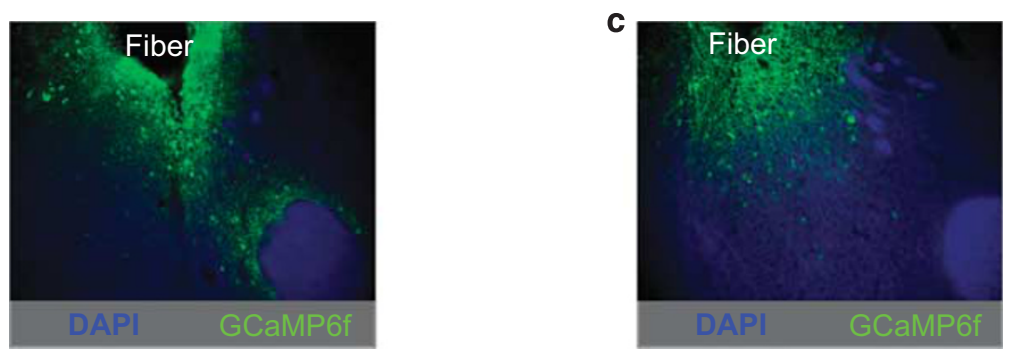

d

D1 Pre-Defeat Time in IZ

e

D2 Pre-Defeat Time in IZ
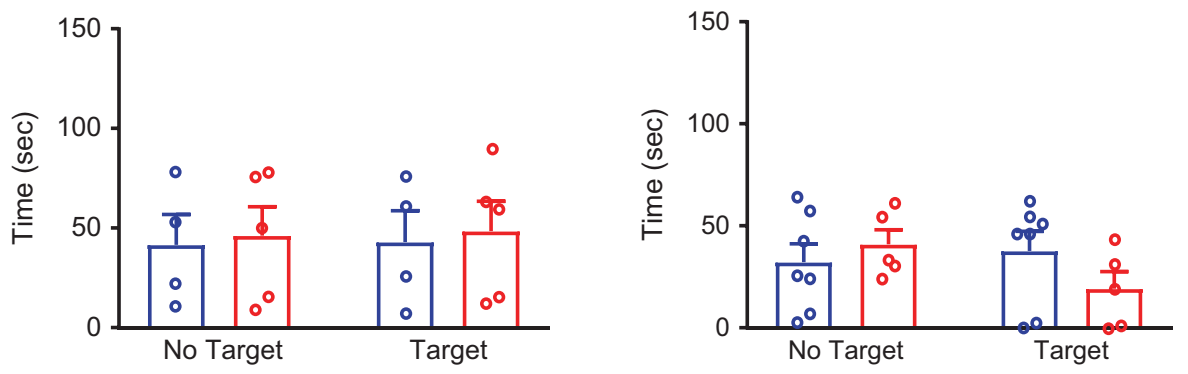

$\square$ Resilient

$\square$ Susceptible

D1 Post-Defeat Time in IZ

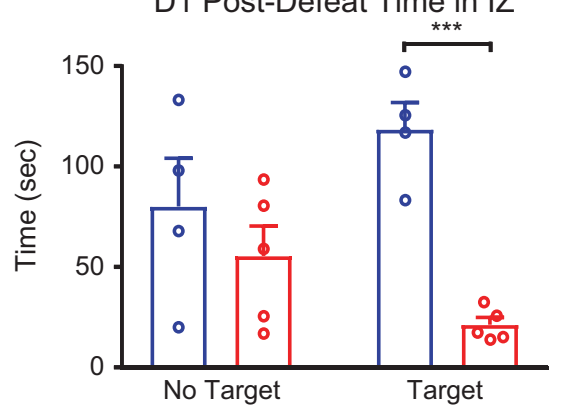

D2 Post-Defeat Time in IZ

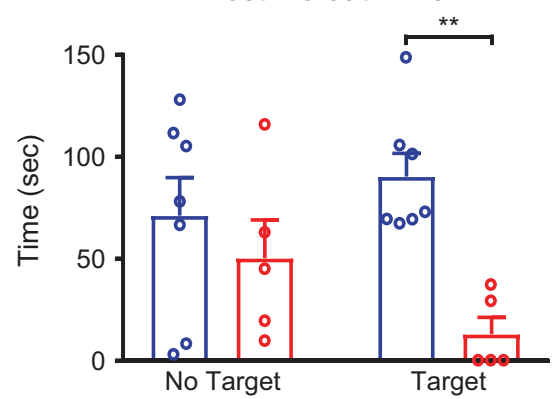

Figure I In vivo fiber photometry imaging to identify latent mechanisms of susceptibility and resilience. (a) Experimental timeline. Immunohistochemistry images show viral GCaMP6f expression and fiber placement in (b) DI-MSNs and (c) D2-MSNs. (d, e) Time spent in the interaction zone during pre-defeat social interaction (SI) test did not differ between groups during either target or no target trials for either DI-cre $(n=4,5)(d)$ or D2-cre $(n=7,5)(e)$ transgenic mice. After CSDS, resilient mice spent more time in the interaction zone during the target trials than susceptible mice for both DI- cre (d) (group effect, $p<0.05$, interaction effect $p<0.05, n=4,5$, post hoc **** $p=0.0008$ ) and D2-cre (group effect, $n=7,5$, post hoc *** $p=0.0046)(e)$ transgenic mice. Data are presented as mean \pm SEM.

Z-score. Peaks were detected as follows: High amplitude events (local maxima two MAD above the median) were filtered out and the median of the resultant trace calculated. The peak detection threshold was set at three times this median and the resultant average peak amplitude and peak frequency were compared across groups. Fiber photometry recordings with no detectable peaks (ie, no significant increases in D1- or D2- MSN activity above baseline) were 
a

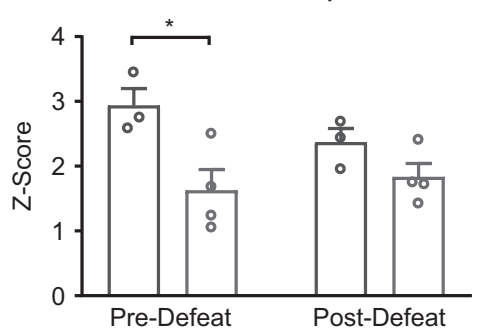

C

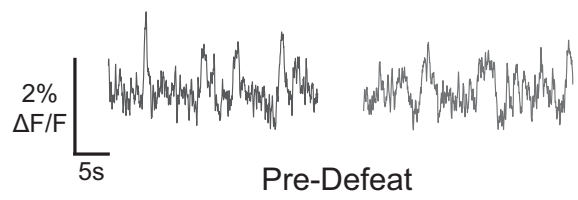

b

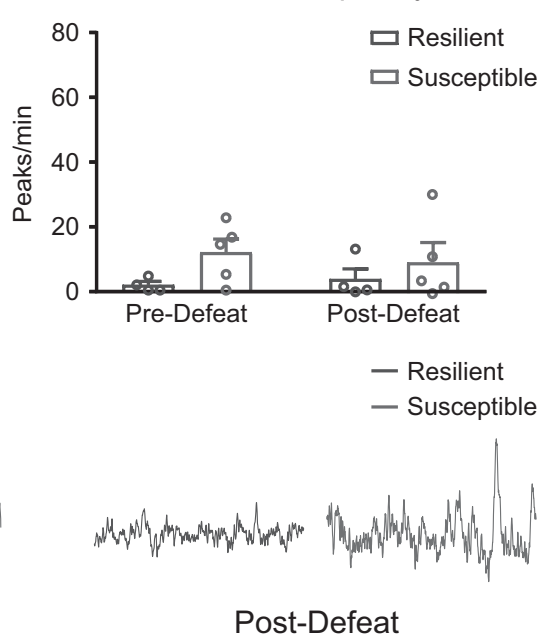

Figure 2 Pre-existing differences in baseline DI-MSN activity associate with later stress phenotype. (a) Amplitude (Group effect, $p<0.05$, post hoc * $p<0.05, n=3,4)$ but not (b) frequency $(n=4,5)$ of calcium transients in DI-MSNs differed between mice that later exhibited resilience vs susceptibility prior to CSDS. (c) Representative 30-s segments of fiber photometry traces. Data are presented as mean \pm SEM.

included in analysis of peak frequency but were not considered in the analysis of average peak amplitude. All animals included in analyses also showed peaks time-locked to behavioral events in at least one of the conditions affirming that recordings without detectable peaks during pre-defeat baseline recordings were not attributable to technical limitations.

For behavior time-locked activity, the maxima within a $5 \mathrm{~s}$ window centered on the behavioral event (corner entry or proximal interaction) were identified in the $Z$-score transformed $\Delta \mathrm{F} / \mathrm{F}$ and the amplitude of these peaks were compared across groups. As the number of proximal interaction and corner entries is different between susceptible and resilient mice, to ensure comparability, only the first incidence of each event was examined. Animals that did not display a particular behavior (ie, proximal interaction or corner entry) were not included in the analysis for that stage of the test (target or no target) but were included in the data for the subsequent stage.

\section{Statistics}

GraphPad Prism 7 was used for statistical analysis. Grubb's test was used to identify and exclude statistical outliers. Twoway repeated measures ANOVA with Sidak's correction for post hoc testing was used.

\section{RESULTS}

Pre-Existing Baseline Differences in D1- but not D2MSNs Associate with Post-Defeat Stress Phenotype

To record cell-specific activity in awake behaving mice, we injected AAVdj-EF1 $\alpha$-DIO-GCaMP6f-WPRE into the NAc of D1- and D2-Cre transgenic mice and recorded $\mathrm{Ca}^{+2}$ transients in a population of infected cells through an implanted optic fiber prior to and after the first aggressive encounter (Figures 1a-c). Naive mice were tested in a social interaction test prior to any stress manipulations and were later defined as resilient or susceptible based on time spent interacting with a social target in a SI test $24 \mathrm{~h}$ after ten days of social defeat (resilient mice $>60 \mathrm{~s}$ in interaction zone with target, susceptible, $<60 \mathrm{~s}$ ). Pre-defeat, time in the interaction zone did not differ (Figures 1d and e) between mice that would become susceptible or resilient after defeat. Post defeat, resilient mice spent significantly more time in the interaction zone than susceptible mice when the target was present, an effect seen in both D1-cre $\left(\mathrm{F}_{(1,14)}=16.84\right.$, $p=0.0011, \quad \mathrm{~F}_{(1,14)}=5.96, \quad p=0.0285, \quad n=4,5$, post hoc $\left.{ }_{* * *} p=0.0008\right)$ and D2-cre transgenic mouse lines $\left(\mathrm{F}_{(1,20)}=9.86, p=0.0052, n=7,5\right.$, post hoc $\left.{ }^{* *} p=0.0046\right)$. Although mice that would become susceptible or resilient did not differ behaviorally prior to CSDS, we nevertheless observed differences in neuronal activity prior to defeat. In neuronal activity recordings immediately before the first aggressive encounter, D1-MSN baseline peak amplitude was larger in mice that later became resilient than in mice that later became susceptible $\left(\mathrm{F}_{(1,5)}=7.56, p=0.0404, n=3,4\right.$, pre-defeat post hoc, $p=0.0123$; Figures $2 \mathrm{a}$ and $c$ ); this difference was not significant in recordings immediately after the first aggressive encounter (post-defeat post hoc $p=0.3412$ ). The frequency of peaks was not different between groups (Figures $2 \mathrm{~b}$ and $\mathrm{c}$ ). In contrast to D1-MSNs, pre-defeat baseline peak amplitude in D2-MSNs did not differ between groups (Figures $3 \mathrm{a}$ and $\mathrm{c}$ ). Although the frequency of peaks was increased in mice that later became susceptible when averaging across pre- and post-defeat recordings, this effect did not reach statistical significance when assessed by individual post hoc tests at either pre- or post- defeat time-points $\left(\mathrm{F}_{(1,9)}=8.69, p=0.0163, n=6,5\right)$ (Figures $3 \mathrm{~b}$ and $\mathrm{c}$ ). These results suggest that lower D1-MSN 
a D2-MSN: Amplitude

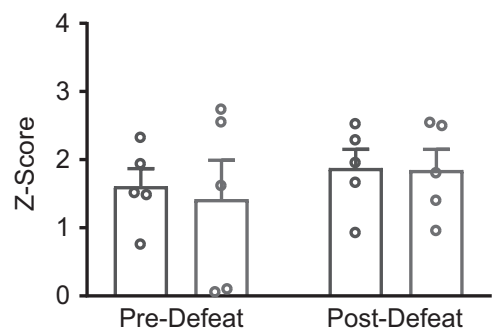

C

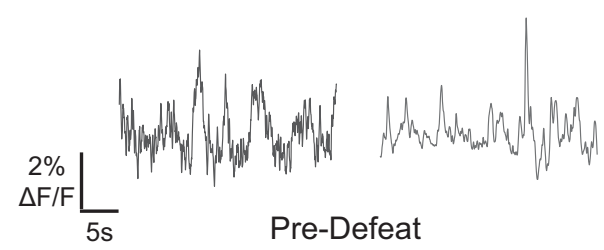

b

D2-MSN: Frequency

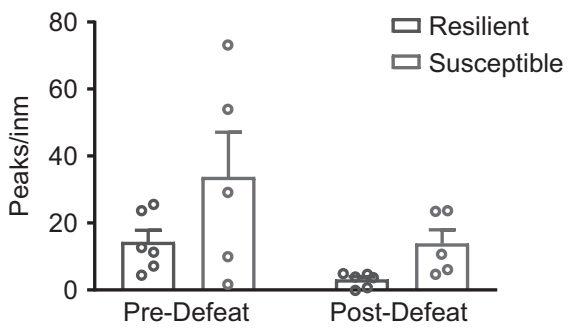

- Resilient

- Susceptible

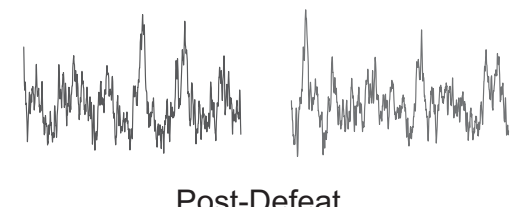

Figure 3 Baseline D2-MSN activity does not associate with later stress phenotype. No differences in (a) amplitude $(n=5,5)$ or $(b)$ frequency (group effect, $p<0.05$, post hoc tests non-significant, $n=6,5$ ) of calcium transients were detected in D2-MSNs during baseline home-cage recordings prior to CSDS. (c) Representative 30-s segments of fiber photometry traces. Data are presented as mean \pm SEM.

activity precedes the behavioral state of susceptibility that is revealed after chronic stress.

\section{Pre-defeat D1-MSN Activity in Resilient but not Susceptible Mice is Specific to Social Interaction}

Having identified baseline differences in D1-MSN activity, we then asked if temporally specific signaling of NAc MSNs associated with specific behaviors during social interaction prior to CSDS (Figure 1a). Previous work identified increased activity in the ventral tegmental area (VTA) during bouts of interaction with a novel mouse that predicted the onset of social behavior, with activation of VTA-NAc projections favoring social behavior (Gunaydin et $a l, 2014)$. To determine whether pre-defeat D1-MSN activity associates with later CSDS-induced behavioral states, we examined peaks in activity that were time-locked with behavioral events including direct social (proximal) interaction and corner entries and then compared the amplitude of these time-locked peaks across groups (Figure 4). Similar to previous findings in VTA, we found that the largest timelocked peaks in D1-MSN activity occurred during social interaction (Figure 4a), although we also observed smaller peaks associated with corner entry (Figure $4 \mathrm{~b}$ ). Between group comparisons revealed that proximal interaction peaks were larger than corner peaks in mice that later exhibited resilience, but not susceptibility, when a social target was present and that proximal interaction peaks in the presence of the social target were larger in future resilient than susceptible mice (Figures $4 \mathrm{c}$ and e; $\mathrm{F}(1,7)=15.87, p=0.0053$, $n=4,5$, post hoc $\left.{ }^{* *} p<0.0077,{ }^{*} p<0.0346\right)$. However, peaks did not differ in either susceptible or resilient mice in the absence of the social target, nor was the amplitude of peaks different between susceptible and resilient mice (Figures $4 \mathrm{~d}$ and f). Thus, we observed that increases in D1-MSN signaling are specifically associated with social interaction in mice that will become resilient, again pointing to a role for pre-stress D1-MSN signaling in determining the outcome of stress-induced adaptations.

\section{Temporally Associated D2-MSN Activity is not Modulated by Social Interaction}

We then compared peaks in D2-MSN activity time-locked with behavioral events (Figures $5 \mathrm{a}$ and $\mathrm{b}$ ). As with D1-MSNs, we observed peaks during social interaction and corner entries (Figure 5a). In contrast to D1-MSN signaling, the amplitude of D2-MSN events did not differentiate proximal interaction and corner entry events in the presence of the social target (Figure $5 \mathrm{c}$ and e). However, in the absence of the target, D2-MSN peaks in resilient mice were smaller during proximal interaction events compared to corner events $\left(\mathrm{F}_{(1,9)}=13.53, p=0.0051, n=6,5\right.$, post hoc, ${ }^{* *} p=0.0049$; Figure $5 \mathrm{~d}$ and $\mathrm{f})$. We observed no differences in susceptible mice. These data suggest that D2-MSNs may convey differential information in resilient compared to susceptible mice but this signal is not specific to social interaction.

\section{DISCUSSION}

Identifying the endogenous mechanisms that lead some individuals to become susceptible to stress and others resilient is essential for developing effective treatments and, ultimately, preventing the emergence of depression. Here, we identified pre-existing differences in baseline D1-MSN activity that associate with the subsequent development of stress susceptibility. Although much previous work has examined how activity in brain reward areas is altered by stress (Bagot et al, 2015; Chaudhury et al, 2013; Christoffel et al, 2015; Francis et al, 2015; Gunaydin et al, 2014; Lim et al, 2012; Tye et al, 2013), very little is known about the root cause of stress susceptibility. We report that, prior to encountering stress, mice that will go on to become resilient already have higher baseline D1-MSN activity as compared 
a
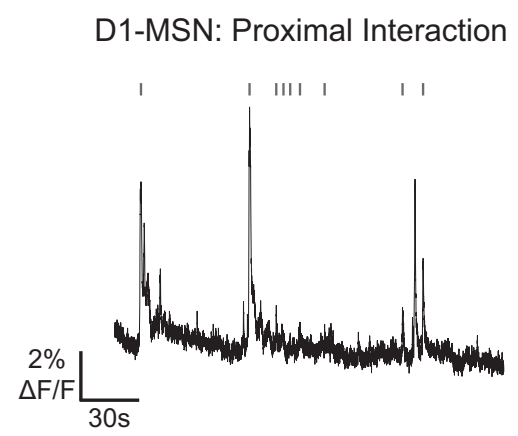

C

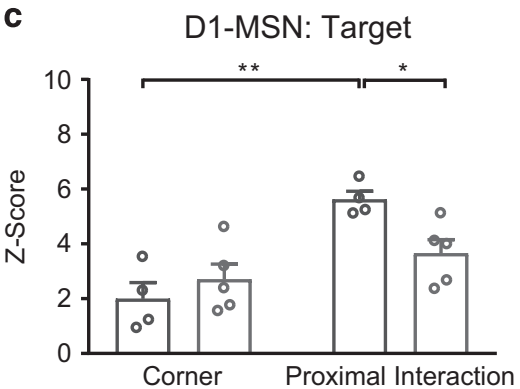

D1-MSN: Target
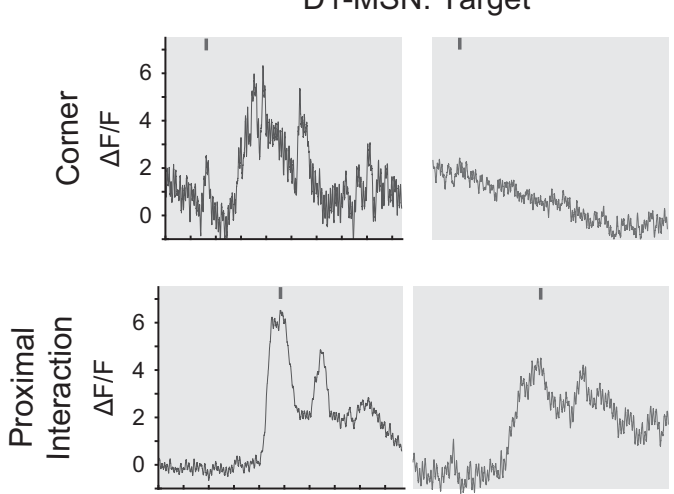

b

D1-MSN: Corner

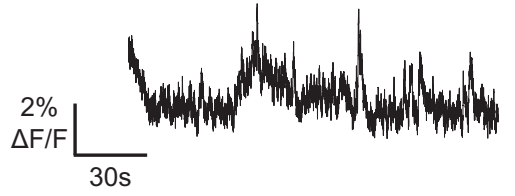

d

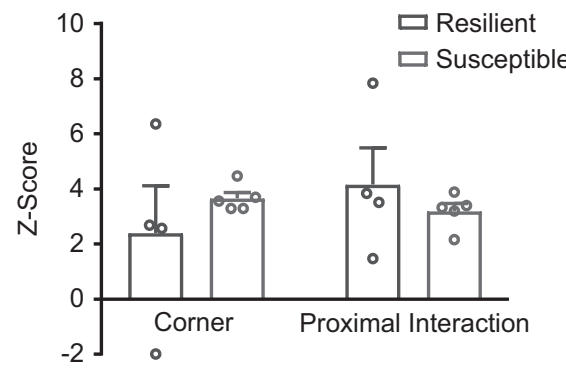

f

D1-MSN: No Target
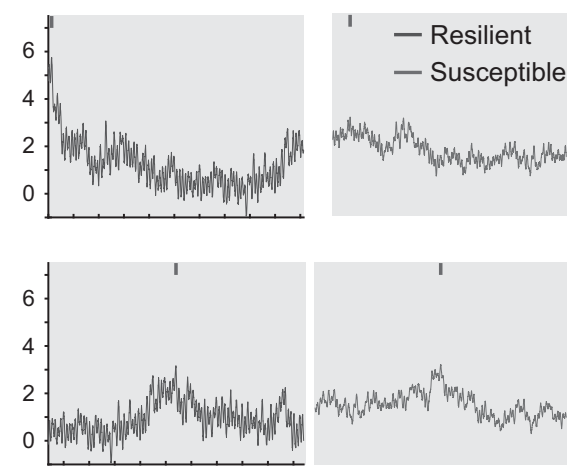

Figure 4 Pre-defeat DI-MSN activity temporally correlated with social interaction is increased in future resilient mice. Photometry traces from mice during a pre-defeat social interaction (SI) target test show large increases in calcium transients in DI-MSNs during SI (proximal interaction with target) (a) and smaller increases during corner entries (b) (red dashes at top of panel indicate occurrence of behavioral events). (c) When a target was present, peak amplitude of calcium transients differed between future resilient and susceptible mice during the first proximal interaction event, and between the first corner entry and first proximal interaction event in resilient mice (effect of event $p<0.05$, post hoc $*<<0.05$, $* * *<0.05, n=4,5$ ). (d) In the absence of the target, peak amplitude of calcium transients during first proximal interaction and corner entry event did not differ between groups $(n=4,5)$. Representative I0-s segments of fiber photometry traces aligned with first corner entry (upper panel) or first proximal interaction (lower panel) during target (e) or no target (f) tests. Data are presented as mean \pm SEM. A full color version of this figure is available at the Neuropsychopharmacology journal online.

with mice that go on to become susceptible. In contrast, we observed no baseline differences in D2-MSN activity. Further, we find that D1-MSN, but not D2-MSN, signaling is specific to social interaction in resilient, but not susceptible, mice. Together, these data highlight that preexisting reward circuit dysfunction is a critical mediator of depression vulnerability.

Fiber photometry provides a population recording of calcium transients and as such the precise interpretation of differences in peak amplitude and frequency are somewhat complex. An increase in peak amplitude could indicate either an increase in the number of cells firing or in increase in the synchronicity of firing from a constant population of cells, whereas an increase in peak frequency could reflect an increase in the number of cells firing, an increase in the frequency of firing of the same number of cells or less synchronous firing of the same number of cells firing at the same frequency. In the present study we found an increase in peak amplitude with no significant change in peak frequency for D1-MSNs. One possible, but not the only, interpretation of our data is that the number of D1-MSNs firing at baseline is increased in mice that go on to become resilient. Although we cannot definitively conclude the precise nature of the activity change in D1-MSNs from population recordings, such data satisfies our primary goal to identify a cell-specific signature of stress susceptibility, despite a certain degree of ambiguity inherent in population recordings. It would be interesting to pursue this finding in a follow-up study focusing on single cell activity in vivo with alternative calcium imaging techniques. 
a D2-MSN: Proximal Interaction

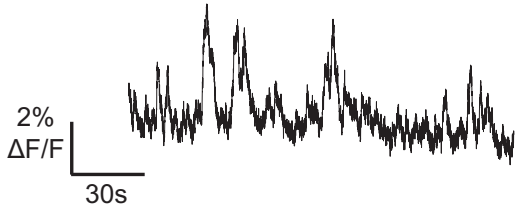

C

D2-MSN: Target

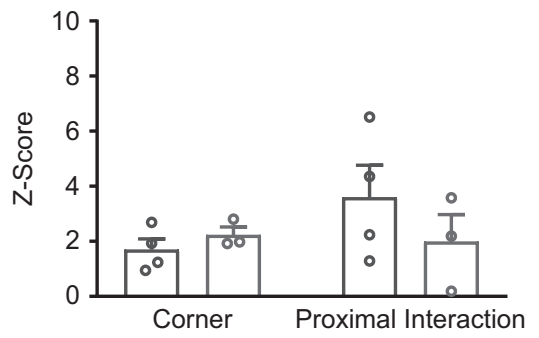

e
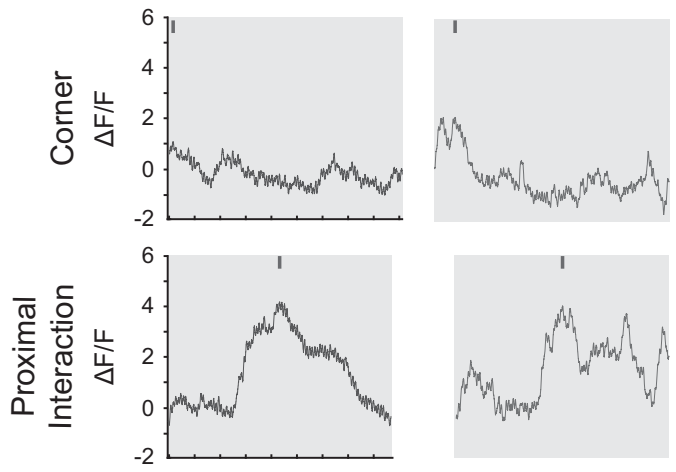

b
D2-MSN: Corner
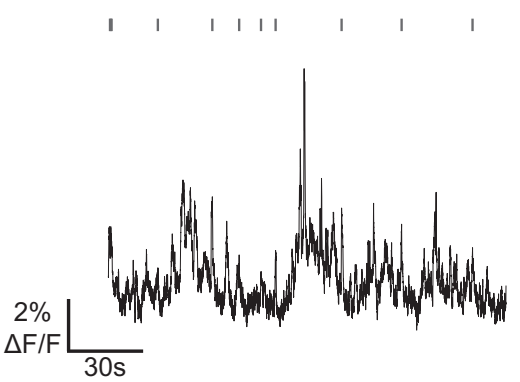

d

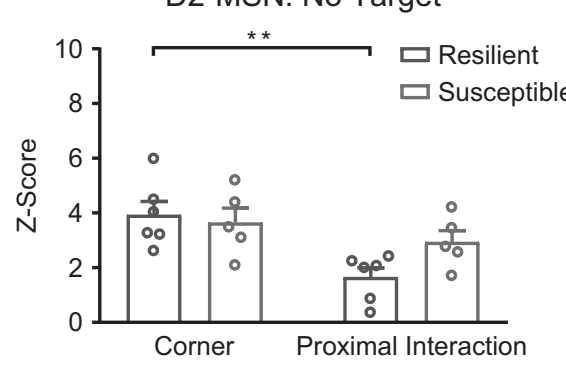

f
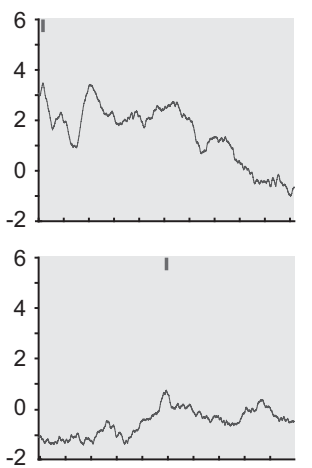

Figure 5 Pre-defeat D2-MSN activity varies with behavioral event. Photometry traces from mice during a pre-defeat social interaction (SI) test show increased calcium transients in D2-MSNs during SI (proximal interaction with target) (a) and comer entries (b) (red dashes at top of panel indicate occurrence of behavioral events). (c) When a target was present, peak amplitude of calcium transients during the first proximal interaction and first corner entry did not differ between groups $(n=4,3)$. (d) In the absence of the target, peak amplitude of calcium transients was lower for resilient mice during proximal interaction compared to corner entry (effect of event, $p<0.05$, post hoc, ${ }^{*} * p<0.05, n=6,5$ ). Representative I0-s segments of fiber photometry traces aligned with first corner entry (upper panel) or first proximal interaction (lower panel) during target (e) or no target ( $\mathrm{f}$ ) tests. Data are presented as mean $\pm \mathrm{SEM}$. A full color version of this figure is available at the Neuropsychopharmacology journal online.

Previous studies of stress-induced changes in NAc activity also point to a specific role for D1-MSNs in mediating resilience to stress (Francis et al, 2015; Lim et al, 2012; Lobo et al, 2013). Artificially increasing D1-MSN activity through optogenetic manipulations after defeat was pro-resilient and pharmacological inhibition of these cells increased susceptibility (Francis et al, 2015). In contrast, manipulating D2-MSN activity after defeat did not influence susceptibility. Moreover, after CSDS, excitatory input to D1-MSNs in susceptible mice was reduced relative to stress-naive mice, an effect not observed in resilient mice (Francis et al, 2015). Decreased synaptic strength was also observed in D1-MSN in mice exhibiting anhedonia after chronic restraint stress (Lim et al, 2012). Our findings suggest that these changes observed after stress may in part reflect the pre-existing increases in D1-MSN activity that we identify in vivo in stress-naive mice during social interaction. We suggest that, similar to the proresilient effect of optogenetic activation of D1-MSNs (Francis et al, 2015), enhanced intrinsic activity prior to defeat may buffer against the deleterious effects of stress, rendering mice resilient. Taken together with previous evidence of poststress alterations in neuronal activity, we conclude that pre-existing individual differences in D1-MSNs have an important role in determining later stress susceptibility with increased activity promoting resilience to chronic stress.

Intriguingly, the magnitude of temporally specific D1-MSN activity differentiated social interaction from corner events only in resilient mice. This finding suggests that, even in the absence of pre-defeat behavioral differences, social interaction may be processed differently at the neuronal level prior to stress in mice predisposed to be resilient $v s$ susceptible after chronic stress. Previous work 
showed that activity of VTA dopamine (DA) neurons projecting to NAc is increased during social interaction and that activation of D1-MSNs, but not D2-MSNs, is necessary and sufficient to initiate social interaction (Gunaydin et al, 2014). Profiling the endogenous signaling of D1- and D2-MSNs suggests that this basic mechanism may be compromised in mice that become susceptible. D1MSN activity in susceptible mice was not different between interacting with the target mouse and corner entry. Potentially, this lack of differential D1-MSN activation could suggest that social interaction is processed differently in susceptible mice even before they encounter stress. Although the mechanism underlying this difference in susceptible mice remains unknown, it is not genetic given that we are utilizing genetically inbred mice, despite the fact that genetic factors are also known to contribute to differences in stress responses among outbred populations.

Dopaminergic projections from the VTA provide important modulatory input to NAc MSNs (Baik, 2013) and previous work has identified a role for VTA-NAc projections in regulating stress susceptibility. Following chronic mild stress (CMS), optogenetic VTA DA activation reverses depressive-like symptoms, an effect dependent upon NAc DA receptors. Conversely, inhibition of VTA DA neurons causes depressive-like symptoms and CMS reduces VTA activity (Tye et al, 2013). In contrast, after CSDS, increased firing of VTA DA neurons is observed specifically in susceptible mice. Moreover, bulk activation of VTA DA neurons or targeted activation of NAc-projecting VTA DA neurons is pro-susceptible (Chaudhury et al, 2013). Although these findings appear contradictory, mild and more severe forms of stress have been shown to exert different effects on VTA DA firing (Valenti et al, 2012). It is possible that the balance between VTA projections to D1- and D2-MSNs may be differentially affected by different stressors and may also have a role in regulating future stress susceptibility. Although the clearest effects we observed were in D1-MSN signaling, we also found modest differences in D2-MSN signaling, although these did not associate with social interaction behavior. Our findings suggest that, pre-stress, mice that will become resilient may already have increased DA input to D1-MSNs as evidenced by increased neuronal activity during social interaction. Integrating our findings with published data, we speculate that CSDS may decrease VTA input to D1-MSNs to drive depression-like states and, that in mice which become resilient, pre-existing increases in VTA input to D1-MSNs mitigate against this later pro-susceptible stressinduced plasticity. However, it is important to note that the pro-susceptible effect of enhanced VTA-NAc activity during CSDS may be mediated by brain-derived neurotrophic factor (BDNF) rather than DA, with BDNF effects predominating on D2-MSNs(Wook Koo et al, 2016). This underscores the complexity of factors that control NAc MSN activity under normal and stressful conditions, and emphasizes the need for future work to identify the specific inputs onto D1-MSNs that are responsible for their enhanced baseline activity in a social setting in mice that are inherently resilient.

Overall, we conclude that pre-existing differences in D1MSN activity are an important determinant of resilience. Our findings offer insight into the mechanistic basis of differential adaptation to stress and hold promise for identifying those individuals most at risk of becoming susceptible in the future, prior to encountering significant life stress. The incidence of depression, and the associated costs to affected individuals and society, continue to increase. Identifying neuronal signatures of risk prior to the emergence of observable behavioral change will open the door to targeted treatments for at-risk groups and, thus, the potential to address the underlying pathology even before the disorder emerges.

\section{FUNDING AND DISCLOSURE}

This work is supported by a 2014 NARSAD Young Investigator Award from the Brain \& Behavior Research Foundation to RCB. The authors declare no conflict of interest.

\section{REFERENCES}

Bagot RC, Parise EM, Pena CJ, Zhang HX, Maze I, Chaudhury D et al (2015). Ventral hippocampal afferents to the nucleus accumbens regulate susceptibility to depression. Nat Commun 6: 7062.

Baik JH (2013). Dopamine signaling in reward-related behaviors. Front Neural Circuits 7: 152.

Berton O, McClung CA, Dileone RJ, Krishnan V, Renthal W, Russo SJ et al (2006). Essential role of BDNF in the mesolimbic dopamine pathway in social defeat stress. Science 311: 864-868.

Calipari ES, Bagot RC, Purushothaman I, Davidson TJ, Yorgason JT, Peña CJ et al (2016). In vivo imaging identifies temporal signature of D1 and D2 medium spiny neurons in cocaine reward. Proce Natl Acad Sci USA 113: 2726-2731.

Chaudhury D, Walsh JJ, Friedman AK, Juarez B, Ku SM, Koo JW et al (2013). Rapid regulation of depression-related behaviours by control of midbrain dopamine neurons. Nature 493: 532-536.

Christoffel DJ, Golden SA, Walsh JJ, Guise KG, Heshmati M, Friedman AK et al (2015). Excitatory transmission at thalamostriatal synapses mediates susceptibility to social stress. Nat Neurosci 18: 962-964.

Covington HE 3rd, Lobo MK, Maze I, Vialou V, Hyman JM, Zaman S et al (2010). Antidepressant effect of optogenetic stimulation of the medial prefrontal cortex. J Neurosci 30: 16082-16090.

Dias C, Feng J, Sun HS, Shao NY, Mazei-Robison MS, Damez-Werno D et al (2014). beta-catenin mediates stress resilience through Dicer1/ microRNA regulation. Nature 516: 51-U86.

Francis TC, Chandra R, Friend DM, Finkel E, Dayrit G, Miranda J et al (2015). Nucleus accumbens medium spiny neuron subtypes mediate depression-related outcomes to social defeat stress. Biol Psychiatry 77: 212-222.

Gerfen CR, Surmeier DJ (2011). Modulation of striatal projection systems by dopamine. Annu Rev Neurosci 34: 441-466.

Golden SA, Covington HE 3rd, Berton O, Russo SJ (2011). A standardized protocol for repeated social defeat stress in mice. Nat Protoc 6: 1183-1191.

Gunaydin LA, Grosenick L, Finkelstein JC, Kauvar IV, Fenno LE, Adhikari A et al (2014). Natural neural projection dynamics underlying social behavior. Cell 157: 1535-1551.

Kendler KS, Karkowski LM, Prescott CA (1999). Causal relationship between stressful life events and the onset of major depression. Am J Psychiatry 156: 837-841.

Kessler RC (1997). The effects of stressful life events on depression. Annu Rev Psychol 48: 191-214.

Kravitz AV, Tye LD, Kreitzer AC (2012). Distinct roles for direct and indirect pathway striatal neurons in reinforcement. Nat Neurosci 15: 816-818.

Krishnan V, Han MH, Graham DL, Berton O, Renthal W, Russo SJ et al (2007). Molecular adaptations underlying susceptibility and 
resistance to social defeat in brain reward regions. Cell 131: 391-404.

Lim BK, Huang KW, Grueter BA, Rothwell PE, Malenka RC (2012). Anhedonia requires MC4R-mediated synaptic adaptations in nucleus accumbens. Nature 487: 183-189.

Lobo MK, Covington HE III, Chaudhury D, Friedman AK, Sun H, Damez-Werno D et al (2010). Cell type-specific loss of BDNF signaling mimics optogenetic control of cocaine reward. Science 330: 385-390.

Lobo MK, Zaman S, Damez-Werno DM, Koo JW, Bagot RC, DiNieri JA et al (2013). DeltaFosB induction in striatal medium spiny neuron subtypes in response to chronic pharmacological, emotional, and optogenetic stimuli. J Neurosci 33: 18381-18395.

Schultz W (2006). Behavioral theories and the neurophysiology of reward. Annu Rev Psychol 57: 87-115.

Sun HS, Damez-Werno DM, Scobie KN, Shao NY, Dias C, Rabkin J et al (2015). ACF chromatin-remodeling complex mediates stressinduced depressive-like behavior. Nat Med 21: 1146-1153.
Tye KM, Mirzabekov JJ, Warden MR, Ferenczi EA, Tsai HC, Finkelstein J et al (2013). Dopamine neurons modulate neural encoding and expression of depression-related behaviour. Nature 493: $537-541$.

Valenti O, Gill KM, Grace AA (2012). Different stressors produce excitation or inhibition of mesolimbic dopamine neuron activity: response alteration by stress pre-exposure. Eur J Neurosci 35: $1312-1321$.

Vialou V, Robison AJ, Laplant QC, Covington HE 3rd, Dietz DM, Ohnishi YN et al (2010). DeltaFosB in brain reward circuits mediates resilience to stress and antidepressant responses. Nat Neurosci 13: 745-752.

Wise RA (2004). Dopamine, learning and motivation. Nat Rev Neurosci 5: 483-494.

Wook Koo J, Labonte B, Engmann O, Calipari ES, Juarez B, Lorsch $\mathrm{Z}$ et al (2016). Essential role of mesolimbic brain-derived neurotrophic factor in chronic social stress-induced depressive behaviors. Biol Psychiatry 80: 469-478. 